CONF-890618--3

DE89 015130

\section{A LASER-DRIVEN SOURCE OF POLARIZED HYDROGEN AND DEUTERUM}

L. Young, R. J. Holt, R. A. Gilman, R. Kowalczyk, K. Coulter

Physics Division, Argonne National Laboratory, Argonne, IL 60439

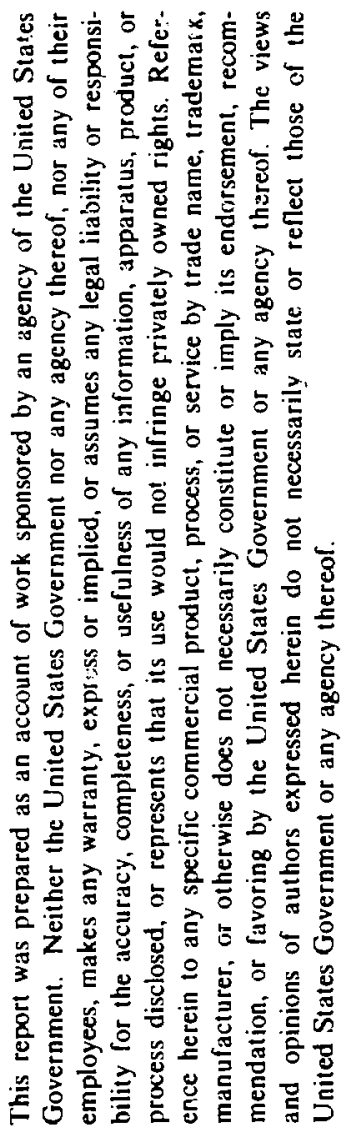

A novel laser-driven polarized source of hydrogen and deuterium which operates on the principle of spin-exchange optical pumping is being developed ${ }^{l}$. This source is designed to operate as an internal target in an electron storage ring for fundamental studies of spin-dependent structure of nuclei ${ }^{23}$. It has the potential to exceed the flux from existing conventional sources $\left.4 \times 10^{16 / s}\right)$ by an order of magnitude. Currently, the source delivers hydrogen at a flux of $8 \times 10^{16}$ atoms/s with an atomic polarization of $24 \%$ and deuterium at 6 $\times 10^{16}$ atoms/s with a polarization of $29 \%$. Technical obstacles which have been overcome, with varying degrees of success, are: 1) complete Dopplei-coverage in the optical-pumping stage without the use of a buffer gas, 2) wall-induced depolarization and 3) radiation-trapping. Future improvements should allow achievement of the design goals of 4 $\times 10^{-7}$ atoms/s with a polarization of $50 \%$.

Fig. 1 shows the prototype laser-driven polarized hydrogen/deuterium source. Two Art-pumped standing-wave dye lasers operating single-mode with Pyridine 2 dye provide the $770 \mathrm{~nm}$ radiation required to optically pump the Dl line of potassium. The spectral density of the lasers is tailored to match the Doppler-broadened absorption profile of the $\mathrm{K}$ vapor $(=1.5 \mathrm{GHz})$ using a $\mathrm{LiTaO}_{3}$ electro-optic modulator configured in a travelling-wave mode as $a \approx 50$ ohm transmission line. The EOM is driven by a series of components which are used to generate high-power white noise. The modulated laser lineshape is characterized by an unbroadened carrier peak superposed on a noise-broadened background. The noise-broadened background has a spectral density which is reasonably well approximated by a Gaussian profile. Typically, the EOMs are nun at a modulation index of 1.5 where roughly $10 \%$ of the power remains in the unbroadened carrier and the bandwidth (HWHM) is determined by the $3 \mathrm{~dB}$ roll-off of the amplifier $(500 \mathrm{MHz})$. The "tailored" laser output is sent through a linear polarizer, circular polarizer, and expanded to fill the cross-sectional area $\left(4.5 \mathrm{~cm}^{2}\right)$ of the spin-exchange cell. At an incident intensity of $=15 \mathrm{~mW} / \mathrm{cm}^{2}$ a factor of ten enhancement is observed in the density of polarized potassium atoms when the laser is noise-modulated. This can be seen in Fig. 2 where the integrated polarization signal (as described below) is increased ten-fold by noise-modulation.

The spin-exchange cell is constructed as an integral unit with the if dissociator (H/D source) and the $\mathrm{K}$ reservoir. It is placed in a static field of $=10 \mathrm{G}$ and heated to $230^{\circ} \mathrm{C}$ to prevent alkali condensation. The interior is coated with a polarization preserving material, drifilms. The flux of the H/D atoms is controlled by a mass flowmeter in conjunction with a servo-driven needle-valve. The density of the $\mathrm{K}$ atoms is independently controlled through the reseryoir temperature. Typically, $K$ densities are $1-3 \times 10^{11} / \mathrm{cm}^{3}$ and $H / D$ densities are $x$ $10^{13} / \mathrm{cm}^{3}$. The usable flux of $\mathrm{HD}$ atoms is determined by measuring, at the output, the fraction of $\mathrm{H}_{2} / \mathrm{D}_{2}$ which remains dissociated using a mechanical chopper, quadrupole mass spectrometer, and lock-in amplifier. A typical fraction for a 1000 bounce cell with a well-prepared drifilm surface is $70-80 \%$.

The polarization is measured by optical detection of magnetic resonance transitions between the various Zeeman sublevels ${ }^{\circ}$. The transparency of the sample to the resonance radiation is decreased by inducing Zeeman transidions with a coil placed at right angles to the holding field. The scheme is shown in Fig.2 and measures polarization for both the alkali and deuterium, assuming a spin-temperature disuibution for the populations of the magnetic sublevels: $N\left(m_{F}\right)=N_{o} \exp \left(\beta m_{F}\right)$. Atomic polarizations of $\geq 80 \%$ are routinely obtained for the alkali, but hydrogen and deuterium polarizations range from $20 \%$ to $40 \%$. 


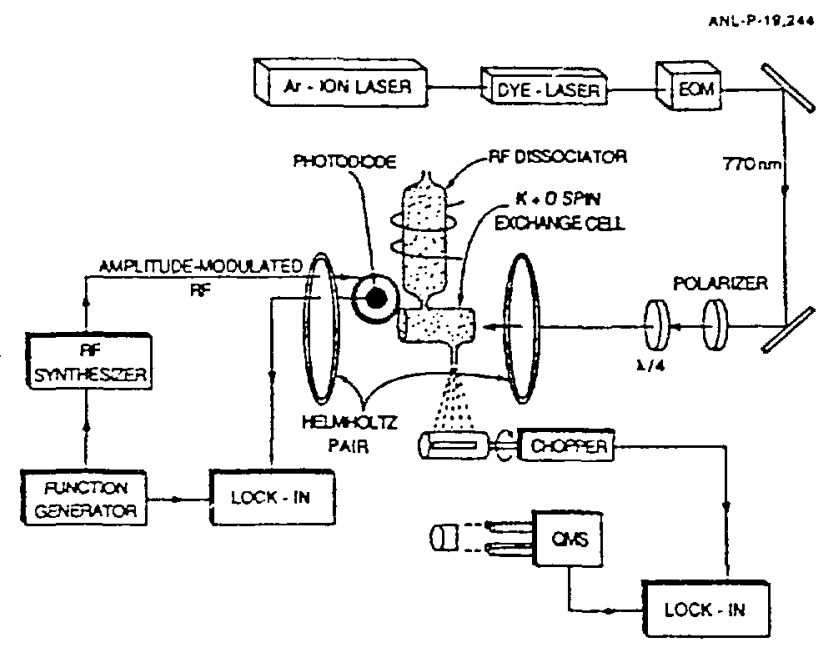

Fig. 1. Schematic of polarized source.

$-$

The foremost challenge in the continued development of this source is to enhance the efficiency of polarization transfer from the alkali to the $H / D$ atoms. A rate equaton model of the polarization transfer process between two spin $1 / 2$ atoms can be used to understand the the relevant parameters? At steady state, the polarization of the H/D, $\mathrm{P}_{\mathrm{D}}$, can be related to that of the alkali, $\mathrm{P}_{\mathrm{A}}$, by $\mathrm{P}_{\mathrm{D}}=\mathrm{P}_{\mathrm{A}} \mathrm{k}_{\mathrm{se}} /\left(\mathrm{k}_{\mathrm{se}}+\mathrm{k}_{\mathrm{d}}\right)$, where $\mathrm{k}_{\mathrm{s}}=\mathrm{n}_{\mathrm{A}} \vee \sigma_{\mathrm{se}}$ is the spin-exchange rate, and $k_{\alpha}=\alpha v(1+1 / a) / 2 a$ is the geometry-dependent wall depolarization rate $(\alpha=$ depolarization

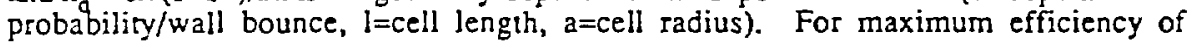
polarization transfer, one should maximize $\mathrm{n}_{\mathrm{A}}$ and $\mathrm{P}_{\mathrm{A}}$ while minimizing $\alpha$.
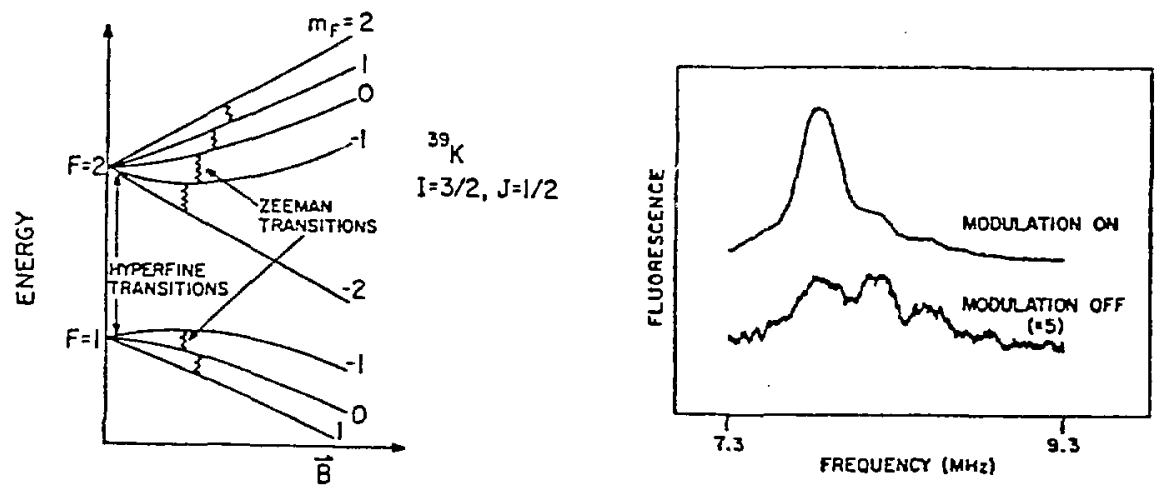

Fig.2. Polarization determination by optical detection of magnetic resonance. Ten-fold enhancement of polarized $\mathrm{K}$ density using noise-modulated laser.

-..-.- - - 
For a given alkali and geometry, the maximum alkali density is limited by radiation trapping. A critical density, $n_{0}$, can be defined as $\left(\sigma_{0} a\right)^{-1}$, where the alkali polatization is degraded to $=50 \%$. Radiation trapping tests on closed cells filled with an $A r$ buffer gas, have shown that the critical density ror $\mathrm{Na}$ is a factor of no greater than that for $\mathrm{K}$, and thus the attainable $\mathrm{k}_{\text {se }}$ using $\mathrm{Na}$ should be two times larger. With this consideration, we have modified the apparatus to use $\mathrm{Na}$, rather than $\mathrm{K}$ as the spin-exchange intermediate. Preliminary results show that even with non-optimal optical pumping conditions for $\mathrm{Na}$ (an estimated $75 \%$ coverage of the Doppler profile) we were able to observe an increase from $\approx 25 \%$ to $\approx 38 \%$ in the deuterium polarization in the spin-exchange cell. Tests with improved laser power and Doppler coverage are curently underway. Future plans include opimization of the cell geometry, using both $\mathrm{K}$ and $\mathrm{Na}$ as spin-exchange intermediates and (if necessary) studies of wall coatings.

\section{References}

1.) R. J. Holt, Proceedings of the Workshop on Polarized Targets in Storage Rings, ANL Repor No. 84-50 (1984); L. Young et al. Nucl. Inst. and Meth. B24/25 963 (1987); L. Young et al., Proceedings of the Topical Conference on Electronuclear Physics with Intemal Targets, SLAC, Stanford CA Jan. 9-12, 1989.

2.) R. J. Holt et al., Nucl. Phys. A446, 389c (1985).

3.) D. H. Beck et al., Proceedings of the 3rd Conference on Intersections Between Particle and Nuclear Physics, Rockpon ME May 14-19, 1988.

4.) H. G. Mathews et al., Nucl. Instr. and Meth. 213, 155 (1983).

5.) D. R. Swenson and L. W. Anderson, Nucl. Instr. and Meth. B28, 627 (1988).

6.) L. W. Anderson and A. T. Ramsey, Phys. Rev. 132, 712 (1963); R. Knize and J. Cecchi, Phys. Lett. 113A, 255 (1985).

7.) T. E. Chupp et al., Phys. Rev. C36, 2244 (1987).

8.) D. Tupa and L. W. Anderson, Phys Rev A36, 2142 (1987).

This research was suppcrted by the U.S. Department of Energy, Office of Basic Energy Sciences, under Contract No. W-31-109-ENG-38. 